\title{
Diagnostic Devices with Microfluidics
}

Edited by

Francesco Piraino • Šeila Selimović

Managing Editor
Krzysztof Iniewski

First published 2017

ISBN: 978-1-4987-7293-8 (hbk)

Chapter 9

Starting with the End in Mind by Developing Diagnostics around User Needs

Mark David Lim

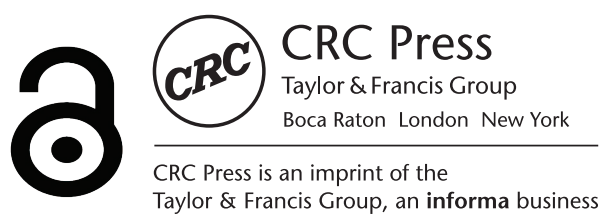





\title{
Starting with the End in Mind by Developing Diagnostics around User Needs
}

\author{
Mark David Lim
}

\section{CONTENTS}

9.1 Defining How the Test Guides a Clinical or Public Health

Decision: Intended-Use .......................................................................208

9.2 Defining How a Test Will Be Used: Use-Cases .......................................2 210

9.3 Defining Where a Test Will Be Used: User-Scenarios ............................214

9.4 Translating Intended-Use, Use-Cases, and Scenarios into Product Requirements...................................................................................... 215

9.5 Translating Product Requirements into Technical Performance

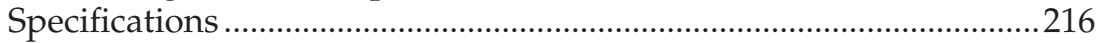

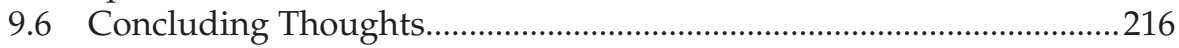

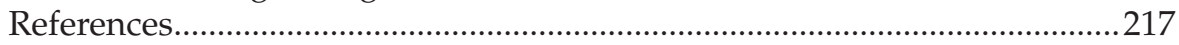

"A couple drops of blood is all that is needed to diagnose a patient's health or disease" is a frequently used headline describing the aspirations for many new diagnostic tools in which both simple collection of small specimen volumes and rapid turn-around-time of test results aim to increase patient compliance and accuracy of treatment. The physician office is often the target market in higher-income countries and private healthcare systems, with recent interest in moving these capabilities closer to the patient in retail settings such as the corner pharmacy. The global health community is another target for these technologies; a healthcare setting is often depicted with images of a minimally trained community health worker who travels extreme distances, solely equipped with a backpack full of drugs and tests administered "under a tree."

Developing diagnostic tools that meet the needs of these settings-a physician office, a pharmacy, and a backpack-seems simple enough. Yet, the fast pace of diagnostics-focused innovations in microfluidics, signal transduction, and multiplexing is faced with an asymmetric adoption rate in any of these settings. Beyond the simplest format, the lateral flow rapid diagnostic test (RDT), and complex cartridges that operate on laboratory-based instruments such as Cepheid's GeneXpert system, there is a paucity of other microfluidic formats that have been implemented at significant scale. 
As discussed in this chapter, the commercialization and adoption bottlenecks for these moderately complex diagnostics cannot be overcome by technological innovation alone, particularly in the highly regulated and payer-limited healthcare and public health markets. Diagnostics, unlike other clinical products, is not an intervention but a decision-aid that guides the use (or nonuse) of an intervention. It is important that the value proposition for any technology-centric innovation in diagnostics include a strong link to a gained efficiency in making a specific decision. Any assay developed without context to the system, users, decision points, and downstream interventions resembles one that is more targeted to the research community, rather than clinical care or public health.

This chapter highlights considerations for designing a diagnostic for use on individual patients or populations by assessing and incorporating the answers to two fundamental questions - who is asking for the test and how do test results guide a decision-questions that cannot be addressed in isolation of the user community. The methodology described is a best-practice for assessing and ensuring that user needs are central to the design, development, and evaluation of a new diagnostic tool. The assessment starts with a clear intendeduse statement that is centered around an actionable decision and justifies the time and cost to obtain a diagnosis. This definition is used to frame use-cases and user scenarios that identify users and describe how the test will be implemented, as well as criteria for generating an actionable test result. All three of these assessments are then used to create a list of product attributes that are required to meet the needs of an end user. The diagnostic developer plays a convening or observer role in the market assessment up until this point but has an active role in defining the technical performance specifications for a product that can satisfy the criteria described in these requirements. This process may be laborious, but centering design principles and functionality around user-needs avoids the creation of a proverbial hammer looking for that nail.

\subsection{Defining How the Test Guides a Clinical or Public Health Decision: Intended-Use}

Oftentimes, the value proposition for a new test is described through improvements in physical operability, speed, and ease of use, with little details on how test results improve a clinical or public health decision. Designing a new diagnostic should always start and remain accountable to a clearly defined "intended-use" that is grounded by the utility of the test's results, framed by an agreed-upon decision algorithm that links a patient encounter with a decision to prescribe or not prescribe a specific intervention (see Box 9.1).

Clinical utility includes circumstance, decision, and action: definitions that justify the use of a test, presentation of test results to a user (e.g., qualitative, 


\section{BOX 9.1 DIAGNOSTIC ALGORITHMS}

The absence of a decision tree or algorithm is a good indicator that the tool is more appropriate for research use instead of the clinical market. It is important to remember that diagnostic tools are used to guide a decision for individual clinical care or population-based public health. For instance, a diagnostic intended for measuring disease prognosis often results in a decision to monitor individuals more or less frequently with another test, depending on the interpretation test results in context of predetermined risk criteria. Similarly, results from a diagnostic used to measure the presence of an infection guide decisions on the course of intervention-using these types of diagnostics without access to treatment options could be considered unethical. Public health diagnostics could be used to monitor the resurgence of a previously quenched disease within a given population; positive results might be weighed with other considerations, such as population mobility, to guide a decision to test each individual with a more specific (and oftentimes more expensive) secondary diagnostic.

These decision trees are often published by a group of disease specialists convened by an overarching organization. Most global health communities, reference guidelines, recommendations, and policies are provided by global or regional departments of the World Health Organization (WHO) and/or by the ministries of health at the country or subnational levels. The algorithms integrate all healthcare toolsdiagnostics and treatment-relying on evidence that assures safety and effectiveness for each recommended decision. Health economics also plays a large part in the rationale for these algorithms in which costeffectiveness includes the resources required to perform the test, test performance, and availability of tools for intervention, considerations that are important for the resource-constrained global health market.

semiquantitative, and quantitative), and types of measurements needed to produce interpretable test results. Defining the patient's situation provides criteria that justifies a decision-maker to administer the test such as presentation of symptoms, ongoing treatment (such as a companion diagnostic), or other risk-associated factors such as geography, environment, or disposition. The action could be prescription or modification of a therapy, additional testing (such as subtyping or confirmatory testing), increased frequency of testing, or a public health action such as quarantine, vector control measure, or mass drug administration.

These attributes - clinical utility, presentation of test results, and interpretation to inform a specific decision-form the two to three sentences that 
comprise an intended-use statement. This statement should match an existing clinical or public health practice, not a hypothetical or aspirational workflow, as these also describe the targeted commercial market and regulatory pathway. It is an uphill battle if a developer envisions that a new technology will change guidelines and workflows because of placement of a new technology in a clinical care or public health program.

In addition to identifying the market, the intended-use statement is central to the design, development, and evaluation of a diagnostic by separating "nice-to-have" technical attributes from "must haves." The latter are the bare minimum requirements to successfully meet the needs of an end user in which every claim in the intended-use statement needs to be validated with clinical evidence. One should be critical of these intended-use claims, remembering that analytical validation studies are much simpler to perform and demonstrate compared to clinical validation studies. For example, if quantitative test results are essential for guiding a decision, studies can demonstrate analytical feasibility using spiked or contrived specimens. However, clinical feasibility must be demonstrated using nonaltered specimen from patient populations representative of the actual intended use, with statistically structured studies that evaluate the ability of the test to reproducibly provide quantitative results relevant to the decision. Challenges could include access to specimens annotated with this level of quantitative data as well as lack of a quantitative gold standard comparator, and it is important to assess the availability of these resources during the planning process of the project. Alternatively, the bar for validating a test's ability to reliably provide a qualitative "yes/no" result may be easier to cross, particularly if this type of test result is all that is needed to guide a specific decision; in this example, quantitation may be a "nice-to-have" that does not impact the final decision.

\subsection{Defining How a Test Will Be Used: Use-Cases}

The intended-use statement is a generalizable description that is enriched with use-cases and user-scenarios to add context to the decision algorithm. The goal of this exercise is to systematically identify how a test will be implemented and used, details that define requirements for getting a test used successfully. By weaving together details that identify users, workflows, and the settings in which the test is performed, the use-case and scenarios detail the "needs" that a test should address from the perspective of the user(s) within a defined healthcare system or public health program.

It is important to note that each use-case does not equate to a single independent test; one diagnostic tool can solve the needs of many use-cases. However, a test developer should demonstrate feasibility against one use-case and then consider expanding to other use-cases. It would be detrimental to develop a 
diagnostic that is not accountable to an intended use and associated use-case, or resource-intensive to simultaneously address multiple use-cases.

As mentioned earlier, a use-case is not developed by a test developer as these descriptions are inherently technology agnostic and should be developed by a community of end users to reflect current clinical or public health practice. At a high level, use-cases include details specific to the targeted patient population, decision-maker (user), healthcare or public health system, and linkage between the specific decision and interpretation of test results.

Use-cases always start with identifying the patient population targeted by the diagnostic and expand on the short description used in the intendeduse statement. These include patient characteristics that trigger the use of a test by a decision-maker, such as symptoms, ongoing treatment, associated risk factors, etc. Additional details include potential coinfections or other factors that define eligibility or ineligibility for a test such as behavior, lifestyle, geography, environmental exposure, etc.

It is also important to understand the patient situation. For example, some patients and their families might have made substantial effort to seek healthcare and thus may resist the concept that a test will determine if they receive treatment, particularly if the testing adds out-of-pocket cost or time. It is also important to understand the role of culture for a specific patient population - a positive test result may result in stigma or isolation, rather than seeking care. If properly identified, many of these risks can be mitigated in the design or implementation of a test. For instance, there may be different ease-of-use considerations if a tribal healer is more effective at obtaining patient compliance for testing compared to a healthcare worker. It may also be important that testing staff include resources for proper counseling if a patient will be informed about their test results.

Another component of the use-case is the identity of the user, the individual responsible for making the clinical or public health decision with authority and resources to act on the test results, in accordance to a decision algorithm. By identifying the user, one can also deduce their workload. For instance, a clinician with a significant patient load might prefer prescribing treatment rather than waiting for test results. The user should not be confused with the test operator who performs the test, a role that is defined in the user scenarios.

These details include circumstances for "why" the user seeks the test results, "what" decisions are made by the user, and "how" test results guide their decision. In many public health programs, the user may be a program manager who is only interested in aggregated diagnostic results of a population, whereas a clinician or healthcare worker may be interested in the test results of an individual patient. Details may also include incentives or challenges for administering the test, such as cost or program achievements. Designing a test that addresses the pressures, incentives, and circumstance of the user are important not only for adoption by a healthcare or public health 
system, but also routine use. Table 9.1 lists some assumptions about the user that should be defined in a use-case.

Use-cases also include how the test will be interpreted, framing the criteria for making the results meaningful to the decision faced by the user. These details provide context to the decision algorithm by describing the type of analysis (e.g., organisms/molecules) and measurement parameters necessary for making a decision (e.g., level of quantitation). Natural history of disease can also be included if aspects such as stage or progression of disease are essential considerations for making a decision. This component of the use-case should also include integration with other data sources if the decision requires consideration of nondiagnostic data.

Severity of risk to a patient's health from an inaccurate result or diagnosis should also be elucidated, as related to the unintended misuse of an intervention. Defining the implications of an inaccurate result to a patient's health sets the threshold for allowable false positive or negative results, which are statistically inevitable but can be mitigated through the use of controls, processes, and other design criteria.

Details about the healthcare system or public health program frame the resources available for administering the test and the prioritization of a specific test in competition with other strategic priorities. It also describes institutional buy-in for using a test, availability and adherence to guidelines or recommendations (see Box 9.1), infrastructure for both administration and sustainable operation of the test, identity of a payer for the test results, and

\section{TABLE 9.1}

Assumptions That Need to Be Evaluated

\begin{tabular}{|c|c|}
\hline Patient & $\begin{array}{l}\text { - Stigma of diagnosis by social circles (family, friends, } \\
\text { community) } \\
\text { - Methods for seeking care (traditional healers, spiritual, } \\
\text { clinical) } \\
\text { - Cultural/spiritual beliefs with collecting specimens } \\
\text { - Privacy considerations for non-blood-based specimens } \\
\text { (urine, stool, genital swabs) }\end{array}$ \\
\hline Community healthcare worker & $\begin{array}{l}\text { - Confidence in operating new technology } \\
\text { - Vulnerability to robbery/theft with more valuable } \\
\text { technology } \\
\text { - Off-label use of technology } \\
\text { - Incentives } \\
\text { - Proficiency testing } \\
\text { - Turnover and training }\end{array}$ \\
\hline Decision maker & $\begin{array}{l}\text { - Patient load } \\
\text { - Incorporation of test into clinical standard-of-care } \\
\text { workflow } \\
\text { - Demographics of patients } \\
\text { - Incentives } \\
\text { - Off-label use of technology } \\
\text { - Proficiency testing }\end{array}$ \\
\hline
\end{tabular}


the regulatory pathway required for implementation. For instance, a public health program focused on a specific global health disease may dedicate resources (infrastructure, labor, funding, supply chain) into a specific testing program and also require that the test is evaluated through the World Health Organization's (WHO) prequalification program ${ }^{1}$ to ensure that the test is manufactured using quality assurance processes and appropriate for use in the global health setting. Conversely, a primary healthcare system may have to balance their services among several disease priorities and may not be able to adequately resource or incentivize the use of a test. These components of the use-case can help forecast and mitigate several potential implementation challenges. Using these examples, a multipathogen platform may be the advantageous route for implementing a diagnostic in the primary healthcare setting but not practical for a disease-focused public health program. Common assumptions that many test developers take are listed in Table 9.2.

\section{TABLE 9.2}

Assumptions about the Healthcare System That Need to Be Evaluated

Lack of testing is due to lack of access/availability of an easily performed test.

Lack of testing is due to high costs of existing test.

Regulatory approval is simpler for global health diagnostics because of the unmet need.
- Identify who recommends the testing of a patient population. Design the performance and usability of a test around a treatment or decision algorithm, which often describes settings for the testing and are described in policy or guidelines authored by a country's Ministry of Health, or at a higher level by the World Health Organization.

- Identify who pays for the test, these usually fall under the auspices of a country's Ministry of Finance or nongovernment procurers such as The Global Fund to fight AIDS, tuberculosis, and malaria.

- Cost of goods should reflect costs and ability to manufacture a design-locked device at scale, not costs reflecting construction of alpha-prototype.

- Costs should not be limited to the test/device by itself, but should include all costs to perform the test. In many instances, costs for training, proficiency testing, and infrastructure to implement a new test may outweigh its technological benefits.

- Components with low cost of goods may not be able to be manufactured at scale or under quality assurance requirements for in vitro diagnostics.

- Diagnostics that guide the clinical course for an individual patient often require the evaluation by a stringent regulatory body and/or prequalification by the World Health Organization.

- Diagnostics that guide public health programs often follow recommendations, guidelines, or policies at the country level, or by the appropriate department of the World Health Organization. 
The details described in this chapter only scratch the surface for developing use-cases, and additional considerations relevant to the implementation of a specific test should be documented as part of the market assessment. As decision-aids, diagnostic tools are an essential part of a healthcare or public health system, and it is important to do these assessments and unpack a list of requirements for integrating a new test into these systems as part of the planning process.

\subsection{Defining Where a Test Will Be Used: User-Scenarios}

A use-case should be further enriched with the depiction of a user-scenario, a series of storyboards that describe "how" a test will be implemented and performed. These storyboards describe the resources needed to perform the test successfully by generalizing the testing workflow. Often a use-case is accompanied by several scenarios, a selection of which represent current practice and others that may require slight changes to the current workflow as necessary compromises for implementing a "better" test. It may be possible to adjust the workflow in small pilot studies, but use of the test broadly and at significant scale requires that the test developer engage the related guideline and payer communities early in the planning process.

This exercise may also reveal that scenarios are dependent on the healthcare systems within a specific geography. By defining as many scenarios as possible, a test developer is given the choice to determine those that align with their business strategy and customize the design of the test around the circumstances of the physical operating environment. As with the use-cases, a test that can be used in all scenarios is likely one that is not perfect for any of them.

At a high level, user scenarios include:

- Patient flow: Mechanism of patient encounter, number of patients seen over a set period of time (hours/days), and workload (patients arrive at one time or over a period of time). These considerations are used to define throughput for the test, batching requirements, and turnaround time for test results. For example, a patient seeking care in a dedicated facility complicates predictions on total throughput, making random access more amenable to this scenario. Conversely, if the patient encounter is through a door-to-door public health campaign, total batch size and turnaround times are dependent on coverage and defined logistics, making these requirements more predictable.

- Testing environment: Examples include testing at the point of contact, transporting a medium-throughput mobile laboratory in proximity to a patient population (e.g., via a van), or collecting the specimens with shipment to a semi/centralized laboratory. These 
considerations are used to define the operational conditions and environments to frame the physical format, availability of resources (running water, electricity, etc.), turnaround time, and accessories necessary for performing the test.

- Staffing: Linked to the patient flow and workflow, this accounts for all individuals who interact with the patient-from preparation of the patient, collection and processing of the specimen, and operating the test. If applicable, it should also identify who provides counsel to the patient on their test results. The education and proficiency for performing these duties should be described as all of these considerations guide ease-of-use and training requirements. These details also inform throughput, batching requirements, turnaround time, and presentation of test results.

- Location of decision-maker (user): Describes whether the decision is made at the same location of the test or if interpretation is made remotely. These considerations are used to define turnaround time and presentation of test results, and data-transmission requirements. For example, a rapid result may not be necessary if, for logistical or cost reasons, the interpretation and intervention options are located in a different location than the test.

- Specimen custody: Details whether the specimen is acquired in a patient's home, in the same location as the test, or is collected remotely and transported to a central laboratory. These details inform the need for additional consumables, such as specimen collection and preservation accessories, and define logistics requirements for batching, shipping, and receiving specimens for testing.

- Test deployment: Resources that a program or healthcare system dedicates to train and prepare test operators and users, assurances for steady supply chain of reagents and equipment maintenance, and infrastructure for transporting test equipment not used in a central location. These details could include procurers, storage, customs regulations, and transportation requirements.

\subsection{Translating Intended-Use, Use-Cases, and Scenarios into Product Requirements}

The last phase of the user assessment is the development of product requirements. This is one of the hardest exercises as it involves compromise between "must have" requirements with "nice to have" attributes. The "must have" product requirements are the minimal criteria to meet end-user needs. They also set the bar for the performance of the test, as the final developed 
prototype must meet all minimum requirements. As with the intended-use, use-cases, and user-scenarios, the product requirements should be defined in collaboration with the end user and technology agnostic.

Compromises are inevitable in the creation of product requirements as they take into context end-user constraints or technical feasibility. For instance, in the global health setting, WHO has defined that the ideal test follows ASSURED criteria (affordable, sensitive, specific, user-friendly, robust and rapid, equipment-free, and deliverable to those who need them). ${ }^{2}$ Each of those criteria have dependencies on each other. For instance, a highly sensitive and specific test may not be affordable or equipment-free, and it is up to the user-community to prioritize these attributes and define them as part of the product requirements.

Product requirements are meant to evolve over time, and it is important to assess any impact of changes to the user scenarios, use-cases, and intended use. It is important to document any change to the original product requirements by their causes and downstream implications to user acceptance.

\subsection{Translating Product Requirements into Technical Performance Specifications}

The product requirements set the goal for any new test, and it is now the responsibility of the developer to create the performance specifications that are achievable using their own technical approaches. This is the step where multiplexing, signal transduction, fluid manipulation, etc., can be incorporated as long as these components improve the ability to meet the product requirements. As mentioned earlier, it may be possible that the original product requirements are difficult to meet with a technical solution, and it is up to the developers to engage the end users to identify implications for compromising these requirements.

\subsection{Concluding Thoughts}

Starting with the end in mind, where end user needs are central to design and development of novel products are the best practices for most sectors outside of biomedicine. As described throughout this chapter, user needs for diagnostics is multifactorial because of the dependencies on both the specific decision and intervention, infrastructure required for implementing the test, and constraints from the regulatory and payer pathways. However, it is important to define criteria for a successful test before designing a new 
diagnostic from the onset because the opposite approach of forcing a technology into the complexities of a healthcare or public health system without this context will likely fail to have meaningful impact.

\section{References}

1. World Health Organization. Prequalification of in vitro diagnostics, http:// www.who.int/diagnostics_laboratory/evaluations/en/, accessed on October 2016.

2. Peeling R.W., Holmes K.K., Mabey A. Sex Transm Infect, 82, 2006, 1-6. 
